\title{
Bienestar psicológico y virtud del coraje en adolescentes tardíos ${ }^{\star 3}$
}

María José Soler

PhD. en Psicología

Universidad de Flores, Argentina Correo electrónico: mariajsoler@gmail.com

\section{Diego García Álvarez}

PhD. Ciencias de la Educación Universidad Dr. Rafael Belloso Chacín, Venezuela Correo electrónico: diegogarcia_123@hotmail.com

Recibido: 20/08/2019

Evaluado: 02/10/2019

Aceptado: 06/11/2019

\section{Resumen}

El objetivo de esta investigación es analizar el bienestar psicológico y la virtud del coraje en la adolescencia tardía, sus posibles relaciones y su diferenciación por sexo. Metodológicamente obedece a un diseño no experimental, transeccional correlacional, con una muestra de 345 adolescentes. Para la recolección de datos se emplearon escalas psicológicas para medir las variables de bienestar psicológico y virtud del coraje, los mismos fueron analizados a través de estadística inferencial no paramétrica. Los resultados son: a) niveles medios de bienestar y virtud del coraje en adolescentes, b) no existen diferencias estadísticamente significativas en bienestar y la virtud del coraje de acuerdo al sexo, y c) correlaciones positivas estadísticamente significativas entre la virtud del coraje y el bienestar psicológico con cada una de sus dimensiones. Los resultados son discutidos desde la perspectiva de la psicología evolutiva de la adolescencia y la psicología positiva.

\section{Palabras clave}

Bienestar psicológico, bienestar del estudiante, virtudes, fortalezas del carácter, virtud del coraje.

3 Para citar este artículo: Soler, M. J. y García-Alvarez, D. (2021). Bienestar psicológico y virtud del coraje en adolescentes tardíos. Informes Psicológicos, 21(2), pp. 43-59 http://dx.doi.org/10.18566/infpsic.v21n2a03

Proyecto de investigación vinculado al Doctorado en Psicología, UFLO. Buenos Aires, Argentina. 


\section{Psychological well-being and virtue of courage in late adolescents}

Abstract

The objective of this research is to analyze psychological well-being and virtue of courage in late adolescence, their possible relationships and their differentiation by sex. The method applied a non-experimental, trans-sectional correlational design, with a sample of 345 adolescents. For data collection, psychological scales were used to measure the variables of psychological wellbeing and virtue of courage. They were analyzed through non-parametric inferential statistics. The results are as follows: a) there are average levels of well-being and virtue of courage in adolescents, b) there are no statistically significant differences in well-being and virtue of courage according to sex, and c) there are statistically significant positive correlations between virtue of courage and psychological well-being with each of their dimensions. The results are discussed from the perspective of developmental psychology of adolescence and positive psychology.

Keywords

\section{Bem-estar psicológico e virtude da coragem em adolescentes tardios}

Resumo

0 objetivo desta pesquisa é analisar o bem-estar psicológico e a virtude da coragem no final da adolescência, suas possíveis relações e sua diferenciação por sexo. Metodologicamente, obedece a um delineamento não experimental, transversal, correlacional, com amostra de 345 adolescentes. Para a coleta de dados, foram utilizadas escalas psicológicas para mensurar as variáveis de bemestar psicológico e virtude da coragem, as quais foram analisadas por meio de estatística inferencial não paramétrica. Os resultados são: a) níveis médios de bem-estar e virtude da coragem em adolescentes, b) não há diferenças estatisticamente significativas no bem-estar e virtude da coragem de acordo com 0 sexo, etc.) correlações positivas estatisticamente significativas entre a virtude de coragem e bem-estar psicológico com cada uma de suas dimensões. Os resultados são discutidos sob a perspectiva da psicologia do desenvolvimento da adolescência e da psicologia positiva.

\section{Palavras chave}

Bem-estar psicológico, bem-estar do aluno, virtudes e forças de caráter, virtude da coragem. 


\section{ntroducción}

La adolescencia es una etapa del ciclo vital que transcurre entre el espacio de la infancia y la adultez joven, es decir, acontece desde el final de la infancia con indicadores biológicos claros de su inicio como la pubescencia y pubertad, pero su final no está claramente definido como etapa evolutiva. De ahí que cabe reflexionar sobre cuáles son los indicadores determinantes que indiquen el comienzo de la adultez joven o emergente, es decir, el cierre de la etapa adolescente (GüemesHidalgo, González-Fierro \& Vicario, 2017). Aunque la psicología evolutiva tradicionalmente ha descrito que la adolescencia es una construcción social en la cual la persona debe alcanzar ciertos indicadores evolutivos y tareas psicosociales, a modo de resumen se tienen: madurez sexual reproductiva, pensamiento hipotético deductivo, formación de la identidad a nivel sexual, vocacional, profesional e incluso ideológica (Papalia, Feldman \& Martorell, 2012).

A pesar de los indicadores evolutivos mencionados anteriormente sobre la trayectoria de la adolescencia como etapa del ciclo vital, cronológicamente se le conoce como adolescencia temprana, media y tardía. Los autores de este trabajo comparten la visión de Vargas (2009) acerca de que la subetapa tardía comprende la resolución de la adolescencia, proceso evolutivo que podría llevarse más tiempo de lo esperado y superar los 1821 años de edad. Tanto Vargas (2009), Güemes-Hidalgo et al. (2017), Amorín (2008), y autores más clásicos de la psicología evolutiva, como Erikson, Kohlberg y Vygotsky (Craig \& Baucum, 2001; Rice, 1997), plantean que algunas áreas del desarrollo, específicamente aquellas relacionadas a la personalidad en sus dimensiones: del carácter, emocional y social, pueden llevar una posible trayectoria distinta de desarrollo cronológico continuo.

En ese orden de ideas, resulta sugestivo describir el bienestar psicológico en la adolescencia tardía, es decir, adolescentes comprendidos desde 17 años a 21 años de edad según la Organización Mundial de la Salud (1995). En ese sentido, desde la perspectiva de Seligman (2011), el bienestar psicológico es un constructo relacional multidimensional basado en cinco elementos con alto grado de valor intrínseco para el sujeto. De alguna forma los siguientes elementos se subjetivizan en la existencia adolescente posibilitando el cultivo del bienestar, a saber: 1) frecuencia de emociones positivas, 2) compromiso y entrega con tareas, 3) relaciones sociales sanas con pares y familiares, 4) percepción de llevar una vida con sentido mediante algún proyecto de vida, y 5) el cumplimento de logros y metas a lo largo de la trayectoria adolescente.

Peterson \& Seligman (2004), dentro del enfoque de la psicología positiva centrada en el estudio del bienestar, proponen que puede verse posibilitado mediante las 6 virtudes humanas conformadas por 24 fortalezas del carácter, es decir, aquellos rasgos con valor moral que hacen que las personas actúen, sientan y piensen como lo hacen. Para estos autores las virtudes y fortalezas del carácter son los ingredientes psicológicos para llevar una vida con bienestar. Vale destacar que la literatura científica ha podido presentar evidencias sobre el rol de las virtudes y algunas 
fortalezas del carácter como rasgos asociados o predictores del bienestar, ajuste social y escolar en la adolescencia, a saber: Brdar \& Kashdan (2010); Giménez (2010); Sol (2012; 2015); Park, Tsukayama, Goodwin, Patrick \& Duckworth (2017); Quinlan, Vella-Brodrick, Gray, \& Swain (2018); Shankland \& Rosset (2016); Wagner (2019); Weber, Wagner, \& Ruch (2016).

Aunque el abanico de las virtudes humanas es amplio conteniendo las virtudes de la sabiduría, coraje, afecto, civismo, templanza y trascendencia, se describirá brevemente sólo la virtud del coraje respondiendo al diseño de la presente investigación. Peterson \& Seligman (2004) explican que la virtud del coraje está conformada por fortalezas emocionales que permiten persistir ante los obstáculos, ser valiente ante situaciones en las que hay que mantenerse a las convicciones personales, ser auténtico en cómo la persona se muestra al exterior, y, por supuesto, el entusiasmo, ilusión y vitalidad en la vida. En otras palabras, la virtud del coraje permite que la persona ejerza su voluntad individual sobre la vida presente y aspectos relacionados al futuro. Sin embargo, a pesar de los antecedentes de estudio mencionados de las fortalezas del carácter, específicamente las fortalezas relacionadas a la virtud del coraje han sido poco estudiadas a nivel mundial, y hay poca evidencia científica de su desarrollo en la adolescencia (Giménez, Vázquez \& Hervás, 2010).

Algunas líneas de investigación han estudiado si existen o no diferencias de acuerdo al sexo en las virtudes humanas y las fortalezas del carácter. En ese sentido, la literatura científica explica que se hallan algunas diferencias en ciertas fortalezas de acuerdo al sexo, pero que éstas no son exageradas (Ovejero, 2010). Investigaciones han referido distintos resultados al diferenciar la virtud del coraje de acuerdo al sexo dentro de sus objetivos específicos, a saber: Ovejero (2010) no encontró diferencias de la variable en relación al sexo, pero Ferragut, Blanca y Ortíz-Tallo (2014a) consiguieron algunas diferencias en las fortalezas de la virtud del coraje, específicamente en las fortalezas de integridad y perseverancia, además de que la virtud del coraje es estable en diferencia a lo largo del tiempo en adolescentes del sexo femenino (Ferragut, Blanca \& Ortíz-Tallo, 2014b). Por su parte, Giménez (2010) sólo halló diferencias significativas de acuerdo al sexo en dos de las fortalezas de la virtud de coraje: mayor valentía e integridad en el sexo femenino.

Siguiendo el orden de ideas, Miljković \& Rijavec (2008) exponen que los jóvenes, en relación a la virtud del coraje, sólo difieren en la fortaleza de la vitalidad. Por su parte, Ovejero y Cardenal (2015) concluyeron que no existen diferencias significativas en las fortalezas de la virtud de acuerdo al sexo, aunque hallaron diferencias pequeñas y moderadas en otras fortalezas de virtudes distintas al coraje, y explican que las hipótesis de su investigación acerca de establecer diferencias en las virtudes y fortalezas del carácter a nivel del sexo no se pudieron verificar, sin embargo, su hipótesis en relación con el coraje era no encontrar diferencias; además, Sol (2015) en una investigación con muestra de niños y niñas argentinos, reportó que no existen diferencias en la virtud del coraje.

En ese sentido, Sánchez-Hernández (2015) explica que sólo existe evidencia 
robusta en la diferenciación de las fortalezas del carácter de acuerdo al sexo en sólo dos grupos de las fortalezas, presentando las mujeres mayores puntajes en las interpersonales, mientras que los hombres en las intelectuales. De igual manera, en relación a establecer diferencias en las fortalezas del carácter de acuerdo al sexo, Ovejero y Cardenal (2015) expresan que las diferencias puntuales en algunas fortalezas pueden ser más explicadas a través de otras variables psicosociales que el sexo en sí como factor determinante.

Por otra parte, la literatura científica ha expresado que posiblemente existan diferencias de acuerdo al sexo en el constructo de bienestar psicológico en la adolescencia, mientras otros estudios no han arrojado diferencias significativas en la variable bienestar y sus dimensiones en hombres y mujeres (Garaigordobil, Aliri \& Fontaneda, 2009), en efecto, no hay acuerdos generales al respecto en la literatura especializada. De igual manera, estos autores exponen que en su investigación sobre bienestar psicológico y otras variables psicológicas en adolescentes españoles, no se hallaron diferencias significativas para hombres y mujeres en bienestar.

En Argentina, Góngora y CastroSolano (2015) exponen que existe mayor presencia de síntomas depresivos y de ansiedad en adolescentes mujeres que en los hombres, así como menor bienestar psicológico. De igual manera, estos autores explican que en su estudio acerca del bienestar psicológico en población argentina con una muestra de 549 sujetos, entre adolescentes y adultos, no se obtuvo diferencias significativas en bienestar en adultos, pero sí en adolescentes masculinos con mayores niveles de bienestar, es decir, estos resultados pueden sugerir trayectorias distintas en el bienestar psicológico de acuerdo al sexo en la adolescencia. Sin embargo, en México, el grupo de investigación de Gómez-Azcarate et al. (2014) informó que no existen diferencias significativas en bienestar psicológico en 1158 adolescentes mexicanos, así como en Uruguay los investigadores García-Álvarez, Soler y Cobo (2019) reportaron no encontrar diferencias en el bienestar psicológico en 188 adolescentes montevideanos.

En ese contexto se formulan las siguientes preguntas orientadoras de la investigación: a) ¿Cuál será el nivel del bienestar psicológico y la virtud del coraje en adolescentes tardíos?; b) ¿Existirán diferencias en el bienestar psicológico y la virtud del coraje de acuerdo al sexo?; y c) ¿Cuál será la relación entre el bienestar psicológico y la virtud del coraje?

De modo que estas formulaciones se operacionalizan en los siguientes objetivos de investigación: a) Caracterizar el bienestar psicológico y la virtud del coraje en adolescentes tardíos; b) Establecer diferencias en bienestar psicológico y la virtud del coraje de acuerdo al sexo; y c) Determinar la magnitud, dirección y significancia de la relación entre el bienestar psicológico y la virtud del coraje. Por lo tanto, el objetivo general de este estudio es analizar el bienestar psicológico y la virtud del coraje en la adolescencia tardía, sus posibles relaciones y su diferenciación por sexo.

Las hipótesis que orientan este estudio descriptivo son las siguientes: a) 
Niveles medios de la presencia de la virtud del coraje y el bienestar psicológicos en adolescentes; b) No se encontrarán diferencias estadísticamente significativas en la virtud del coraje y bienestar psicológico de acuerdo al sexo de adolescentes; c) Se encontrarán correlaciones positivas entre el bienestar psicológico y la virtud del coraje en la adolescencia.

Este artículo busca profundizar sobre el bienestar psicológico como constructo relacional con la virtud del coraje estudiadas en el contexto local montevideano desde la psicología positiva enfocándose en la etapa adolescente tardía. De ahí se esgrime la justificación de la investigación en la dimensión teórica enmarcada en la psicología evolutiva y psicología positiva, en estudiar teorías contemporáneas sobre la concepción del ser humano como ser activo, con rasgos entendidos a modos de virtudes humanas, su posible potenciación y, por supuesto, qué tan pertinentes o no lo son en contexto local. Se busca confirmar que la virtud del coraje aporta un conjunto de fortalezas de personalidad que implica el desarrollo de múltiples hábitos y disposiciones que correlacionarían con los niveles del bienestar en la etapa del ciclo vital adolescente.

A su vez, la justificación metodológica y científica de este estudio está referida a concebir el bienestar psicológico desde una perspectiva multidimensional basado en indicadores de la salud mental y funcionamiento óptimo, alejado de otros estudios realizados en Montevideo en los cuales se estudia el bienestar como ausencia de síntomas de psicopatología a través instrumentos de cribado (Daset, Fernández-Pintos,
Costa-Ball, López-Soler \& Vanderplasschen, 2015; Fitermann \& Zerpa, 2015). Por consiguiente, se busca que esta investigación sea futuro antecedente con datos empíricos en contexto rioplatense para investigadores $y$ profesionales de la salud, psicología y educación.

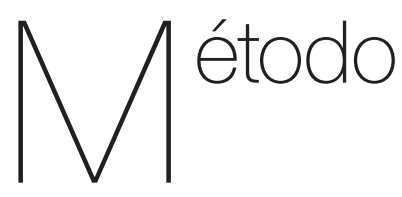

\section{Diseño}

De acuerdo a los objetivos planteados, el diseño de la investigación corresponde al tipo no experimental, descriptivo y correlacional (Hernández, Fernández \& Baptista, 2008).

\section{Participantes}

La muestra de la investigación fue de 345 estudiantes de instituciones preuniversitarias ( $\mathrm{M}=18.98, \mathrm{DE}=1.46)$, comprendidos en la etapa del ciclo vital adolescencia tardía (Organización Mundial de la Salud, 1995), a través de un muestreo no probabilístico de acuerdo a la disponibilidad de los estudiantes en las instituciones que aceptaron colaborar con el presente estudio, específicamente 137 del sexo masculino (39.7\%) y 208 del sexo femenino (60.3\%). La muestra presentó edades comprendidas entre: 17 años (17.1\%), 18 años (24.3\%), 19 años (25.2\%), 20 años (14.8\%) y 21 años (18.6\%). Se destaca que la muestra fue producto de factores de cooperación entre las instituciones educativas con el equipo de investigación. 


\section{Instrumentos de recolección de datos}

Se elaboró un cuadernillo con:

a) Carta de consentimiento informado y descripción de la investigación. Sección de datos socio-demográficos ad hoc.

b) Escala de Bienestar Psicológico para Adolescentes de Soler (2016), escala aditiva de 35 ítems en formato Likert con cinco opciones de respuesta que está basada en el modelo de bienestar psicológico propuesto por Seligman (2011), entendido como un constructo relacional en sus dimensiones de emociones positivas, compromiso, relaciones sociales, vida con sentido y metas. La variable bienestar psicológico se define operacionalmente a partir de la suma de los puntajes obtenidos a través de la respuesta a los ítems que conforman los elementos del mismo, en efecto, a mayor puntuación se interpreta como mayores niveles de bienestar psicológico. La autora sugiere que se calcula la puntuación global de bienestar psicológico, y se toman en cuenta las dimensiones para la interpretación cualitativa, así como presentó evidencias de validez y confiabilidad adecuadas de la escala. La consistencia interna medida a través del alfa de Cronbach para este estudio fue de .905

c) Escala de la Virtud del Coraje para Adolescentes de Soler (2016), basada en la teoría de las virtudes y fortalezas del carácter propuesta por Peterson y Seligman (2004), que consiste en una escala Likert aditiva compuesta por 27 ítems que miden la virtud del coraje a través de cinco opciones de respuesta. La escala está concebida como un instrumento unidimensional que explora la virtud del coraje definida operacionalmente como la puntuación obtenida a través de la suma de las respuestas en cada uno de los ítems, indicando que mayores puntuaciones directas se interpretan como mayor nivel de la virtud del coraje. La escala en el estudio original referido por la autora presentó adecuadas propiedades de validez de contenido, convergente y confiabilidad. La consistencia interna medida a través del alfa de Cronbach para este estudio fue de .718 .

\section{Procedimiento}

La investigación fue diseñada bajo los criterios éticos y legales expuestos por el Ejecutivo Uruguayo (2008) en el Decreto 379/008 y la Asociación Americana de Psicología (APA, 2010) para la investigación psicológica. Asimismo, se contó con la colaboración de varias instituciones educativas a nivel preuniversitario para la recolección de datos en horas libres de los estudiantes. Para empezar la recolección de datos los encuestadores entregaron los cuadernillos de investigación, leyeron el consentimiento informado que cada uno contenía, explicaron el carácter anónimo, voluntario, confidencial y de uso científico de las respuestas. Los estudiantes no recibieron ningún tipo de retribución por participar. Para finalizar, los datos recolectados fueron transcriptos a un procesador estadístico. 


\section{Análisis de datos}

Los datos fueron transcriptos al procesador estadístico IBM SPSS Statistics 25. Primeramente, se realizaron análisis de estadísticas descriptivas enfatizados en medidas de tendencia central, a saber: media, máximo y mínimo. Luego, se procedió a explorar la distribución de las variables mediante la prueba Kolmogorov-Smirnov con la corrección de significación de Lilliefors. Debido al resultado de valores de significancia < .05, se asumió la distribución no normal implicando decisiones prácticas que se materializaron en el uso de la estadística inferencial no paramétrica para la resolución de los objetivos planteados, a saber, la prueba de $U$ de Mann-Whitney de contraste y el coeficiente de correlación Rho de Spearman.

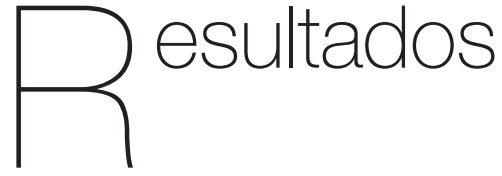

Los resultados serán expuestos en el orden de los objetivos de investigación formulados. En ese sentido, en la Tabla 1 se pueden observar las medias que permiten caracterizar el bienestar psicológico y la virtud del coraje en adolescentes tardíos. Específicamente, la virtud del coraje se ubica en el percentil 50, mientras que el bienestar refiere al percentil 65, es decir, ambas variables están en niveles adecuados en torno a la media estadística.

Tabla 1.

Descriptivos de las variables de estudio

\begin{tabular}{lccccc}
\hline & N & Mínimo & Máximo & Media & Desviación \\
\hline Bienestar psicológico PERMA & 345 & 83.00 & 175.00 & 142.93 & 16.02 \\
P: Emociones positivas & 345 & 18.00 & 40.00 & 30.96 & 5.35 \\
E: Flujo & 345 & 10.00 & 25.00 & 19.11 & 3.28 \\
R: Relacione interpersonales & 345 & 12.00 & 40.00 & 32.14 & 5.29 \\
M: Sentido de vida & 345 & 18.81 & 45.00 & 34.06 & 7.12 \\
A: Metas y logros & 345 & 9.00 & 25.00 & 19.45 & 3.92 \\
Virtud del coraje & 345 & 54.00 & 121.00 & 92.02 & 10.58 \\
\hline
\end{tabular}

Con respecto al objetivo de establecer diferencias en bienestar psicológico y la virtud del coraje de acuerdo al sexo, los resultados obtenidos a través de la prueba de contraste de hipótesis no paramétrica de $U$ de Mann-Whitney muestran que no hay diferencias estadísticamente significativas en las variables del bienestar psicológico ( $z=-1.505, p>$.05) ni en la virtud del coraje $(z=-.399, p>$.05). De igual manera, se encontró que no hay diferencias estadísticamente significativas en ninguna de las dimensiones del bienestar psicológico de acuerdo al sexo, a saber: emociones positivas $(z=-.365, p>$ .05), flujo ( $z=-1.595, p>.05)$, relaciones interpersonales $(z=-2.061, p>.05)$, sentido de vida $(z=-1.686, p>.05)$ y metaslogros $(z=-1.009, p>.05)$, es decir, no se encontraron diferencias significativas 
entre hombres y mujeres para ninguna de las dimensiones estudiadas del bienestar.

Para dar respuesta al último objetivo referido a determinar la magnitud, dirección y significancia de la relación entre el bienestar psicológico y la virtud del coraje, conociendo previamente que las variables no siguen una distribución normal, se llevaron a cabo análisis de correlaciones no paramétricas a través del coeficiente de Spearman. A continuación, en la Tabla 2 se pueden observar los coeficientes de correlación positivos, moderados y significativos interpretados de acuerdo a Prieto \& Muñiz (2000).

Tabla 2.

Correlaciones entre Virtud del Coraje y dimensiones del Bienestar

\begin{tabular}{ccccccc}
\hline Virtud del coraje & Bienestar & $\begin{array}{c}\text { Emociones } \\
\text { positivas }\end{array}$ & Flujo & $\begin{array}{c}\text { Relaciones } \\
\text { interpersonales }\end{array}$ & $\begin{array}{c}\text { Sentido } \\
\text { de vida }\end{array}$ & $\begin{array}{c}\text { Logros y } \\
\text { metas }\end{array}$ \\
\hline Rho de Spearman & $.550^{* *}$ & $.505^{* *}$ & $.418^{* *}$ & $.350^{* *}$ & $.312^{* *}$ & $.497^{* *}$ \\
\hline
\end{tabular}

Nota: $\mathrm{n}=348 ;{ }^{* *}$ La correlación es significativa a nivel .01 (bilateral).

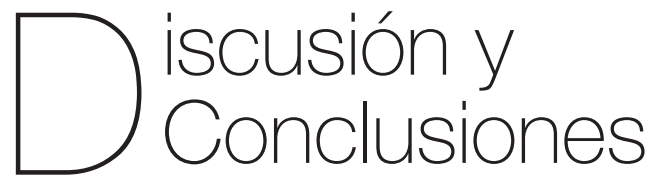

De acuerdo a la teoría del bienestar multidimensional denominada PERMA propuesta por Seligman (2011), se interpreta que la muestra de adolescentes presenta niveles adecuados o medios de bienestar. En ese sentido, se infiere que tienen emociones positivas $(P)$ con frecuencia, experimentan experiencias de flujo (E) entendidas como situaciones de alto nivel de concentración en una tarea específica con apropiada percepción de agencia. Asimismo, se puede deducir que cuentan con apropiados vínculos sociales con sus pares y familiares $(R)$, perciben que su vida tiene cierto sentido expresado en pensar, diseñar y ser consecuentes con un proyecto de vida, en efecto, relacionado con el cumplimento de logros, metas y tareas específicas $(A)$ que se van dibujando dentro de ese proyecto de vida, siendo además acorde a las tareas psicosociales esperadas para esta etapa del ciclo vital.

Desde el enfoque de las virtudes y fortalezas del carácter propuesto por Peterson \& Seligman (2004), se puede interpretar que la muestra de adolescentes presenta nivel medio de la virtud del coraje, es decir, esos rasgos virtuosos que permiten el manejo emocional para persistir en objetivos a pesar de obstáculos, rechazos y trabas en el camino, así como ser fieles a creencias personales sobre las presiones sociales. De modo que la virtud del coraje viabilizaría comportamientos íntegros en el sentido de que las personas sean auténticas, congruentes con sus acciones y honestas en cómo se muestran al mundo externo social. En resumen, presentan comportamientos vinculados a la perseverancia, vitalidad, valentía e integridad. 
De acuerdo al objetivo de establecer diferencias en bienestar psicológico y la virtud del coraje de acuerdo al sexo, no se encontraron diferencias estadísticamente significativas entre hombres y mujeres con relación a ninguna de las variables. Por lo tanto, los resultados concernidos a la virtud del coraje encontrados en esta investigación son congruentes con los antecedentes descritos en la introducción: Ferragut et al. (2014a; 2014b), Giménez (2010), Miljković \& Rijavec (2008), Ovejero (2010), Ovejero y Cardenal (2015), Sol (2015) y SánchezHernández (2015), quienes no encontraron diferencias significativas en la virtud del coraje del acuerdo al sexo.

En relación al bienestar operacionalizado desde la teoría PERMA de Seligman (2011), no se encontraron diferencias significativas entre hombres y mujeres en ninguna de sus dimensiones; estos resultados son congruentes con los antecedentes de investigación reportados por Garaigordobil et al. (2009), Gómez-Azcarate et al. (2014), y García-Álvarez et al. (2019).

Asimismo, los resultados hallados en esta investigación también son congruentes con los de Castellá et al. (2012), quienes realizaron un estudio comparativo del bienestar psicológico en 640 adolescentes entre Argentina y Brasil, encontrando que no existen diferencias significativas en hombres ni mujeres respecto al bienestar. Asimismo, Abdel-Khalek (2014) Ilevó a cabo una investigación con 239 adolescentes libaneses concluyendo que no existen diferencias significativas para bienestar según el sexo. De igual manera, González, Montoya, Casullo y Bernabéu (2002) reportaron que no existen diferencias significativas en bienestar de acuerdo al sexo en 417 adolescentes españoles. Además, García-Andalete (2013) concluyó que no existen diferencias significativas en bienestar en hombres y mujeres estudiantes universitarios españoles, así como Zubieta, Muratori y Fernández (2012) en una muestra de 696 argentinos.

De acuerdo a los resultados orientados a determinar la magnitud, dirección y significancia de la relación entre el bienestar psicológico y la virtud del coraje, se interpretan como congruentes con los planteamientos realizados por Seligman (2003; 2011) tanto en su teoría de la auténtica felicidad y del modelo de bienestar de PERMA, en las cuales el constructo de virtudes y fortalezas del carácter actúa como un eje transversal que directamente cultiva el bienestar psicológico en las personas debido a que son rasgos con cualidades morales que expresan las virtudes del carácter en conductas, pensamientos y emociones. En ese orden de ideas, los resultados de esta investigación son congruentes con aportes de Brdar \& Kashdan (2010); Giménez (2010); Huta \& Hawley (2008); Park, Peterson \& Seligman (2004); Peterson \& Seligman (2004); Shimai, Otake, Park, Peterson \& Seligman (2006); Seligman, Steen, Park \& Peterson (2005) y Sol (2012; 2015) basados en estudiar el rol relacional de las virtudes y fortalezas del carácter en el bienestar psicológico.

Con respecto a la relación entre emociones positivas $(P)$ y la virtud del coraje, se analiza que entre sus fortalezas se halla el ánimo con el que la persona afronta la vida con alegría y entusiasmo haciendo de ésta una aventura que 
merezca la pena vivir. De igual manera, Fredrickson (2009) y Lyubomirsky (2008) plantean que la persona debe contar con la voluntad para construir estados emocionales positivos a pesar de los eventos de la vida, es decir, contar con valentía para realizar acciones desde la voluntad para trabajar por su bienestar, salud mental y crecimiento personal contando con perseverancia para mantenerlo en el tiempo.

Asimismo, la personalidad autotélica (Csikszentmihalyi, 1990) genera emociones positivas a través de la perseverancia para el logro de actividades, ya sean o no gratificantes inmediatamente, y que son logradas manteniendo la motivación por el gusto intrínseco de lograr las metas o actividades que generen flujo. Del mismo modo, estos resultados son congruentes con Sol (2015), específicamente en las correlaciones significativas de la virtud del coraje con el elemento de emociones positivas.

Con base a lo anterior, la relación entre la virtud del coraje y el flow (E) se analiza mediante los criterios necesarios para la vivencia de la experiencia óptima que requieren de alta concentración, compromiso, dominio de la tarea y percepción de reto a las capacidades personales, requisitos que pueden ser vividos desde las fortalezas de la perseverancia, valentía y autenticidad pues éstas presentan aspectos de carácter emocional que ayudan a la persona a afrontar retos o dificultades, terminar las tareas que comienza tolerando obstáculos y aprietos. De igual manera, Sol (2015) reportó correlaciones significativas de todas las fortalezas de la virtud del coraje con el componente flujo y compromiso.
Desde el planteamiento de que la experiencia del flujo se relaciona con el componente de logros y metas en la postura del bienestar de Seligman (2011) con base al enfoque eudaimónico, se comparte la idea de Castro-Solano (2011) quien explica que para el logro de metas se requiere de la voluntad para emplear la inteligencia con fines nobles para conquistar metas virtuosas, el concepto de voluntad es considerado en esta investigación en la virtud del coraje, la cual se expresa en el ejercicio de la misma para alcanzar metas, objetivos y logros (elemento A). Para Seligman (2011) la auténtica felicidad se consigue por el valor intrínseco que tengan esas metas en la vida de la persona, siendo la fortaleza de la autenticidad o integridad de gran relevancia en este aspecto para reconocer qué cosas, eventos y actividades verdaderamente le gustan; además, la persona debe contar con la valentía para asumir esos intereses de manera genuina asumiendo las responsabilidades de sus decisiones. Los resultados son congruentes con los expuestos por Sol (2015) quien halló correlaciones significativas de las fortalezas de la virtud del coraje con el componente de logros y autorrealización.

Por su parte, Duckworth \& Gross (2014) ponen de manifiesto que el Grit (concepto que no tiene traducción al español, propuesto por Duckworth et al., 2007) entendido como rasgo que engloba la pasión y perseverancia por el logro de metas a largo plazo, es un factor esencial pues permite que las persona perseveren, autorregulen y se empujen hacia el éxito. Asimismo, este concepto ha sido medido empíricamente haciendo alusión al elemento de logros y metas de la teoría de bienestar; en efecto, Duckworth \& 
Seligman (2006) plantean que "las personas con mayores niveles de Grit experimentan mayor bienestar en concordancia con que también declaran placeres, engagement y significados en la vida, conformando el elemento vida conseguida" (Seligman, 2011, p. 35) debido a que la persona trabaja de manera perseverante, diligentemente y actuando en defensa de metas a largo plazo que impregnan de sentido a la vida.

En este orden de ideas, la virtud del coraje se correlaciona con la vida con sentido (M) debido a que las fortalezas de la integridad, valentía y perseverancia son necesarias para que la persona pueda desde su autenticidad identificarse con causas que vayan más allá de la propia existencia, así como del valor necesario para defender posturas, causas y visiones de la vida a pesar de los obstáculos, aquí es donde la perseverancia actúa para seguir adelante a pesar de las dificultades. Para Casullo (2005), las fortalezas de la autenticidad y perseverancia son muy importantes para lograr llevar proyectos. La primera relacionada con poder defender creencias sociales y personales que pueden no ser muy bien vistas en un contexto social - histórico determinado, pero son significativas para el bien común. La perseverancia permite que las personas vayan dirigidas al cumplimiento de objetivos que son realmente valorados generando satisfacción y percepción de haber vivido bien la vida. De igual manera, estos resultados son congruentes con los reportados por Sol (2015) quien encontró correlaciones significativas de las fortalezas de la virtud del coraje: vitalidad, autenticidad, perseverancia y valentía con vida con sentido.
Con respecto a la correlación encontrada entre la virtud del coraje y las relaciones interpersonales (R) se interpreta que desde la autenticidad las personas se preocupen, quieran e interesan realmente por otro, en las relaciones humanas son esenciales las características de integridad - autenticidad, pues viabilizan la empatía, liderazgo y asertividad. El coraje como virtud del carácter puede ayudar a conectar relaciones sociales de calidad que se relacionarían con el bienestar psicológico subjetivo, la valentía puede añadir un ingrediente esencial para que la persona pueda desenvolverse en escenarios sociales, para la expresión emocional de cuidado, cariño y hasta de amor. Estos resultados son congruentes con los expuestos por Sol (2015) quien halló correlaciones de la virtud del coraje a nivel significativo con el elemento de relaciones sociales propuesto por Seligman (2011) a excepción de la perseverancia. Por su parte, Park, Peterson \& Sun (2013) han descrito que la fortaleza de la vitalidad se ha correlacionado con relaciones sociales.

Para cerrar este análisis y discusión de resultados, Castro-Solano (2011) llevó a cabo un estudio para detectar cuáles de las virtudes del carácter están relacionadas de manera directa con las tres vías del bienestar psicológico, a saber: vida placentera, vida comprometida y vida con sentido propuestos por Seligman (2003), identificando que la virtud del coraje presenta correlaciones entre todos los elementos en población argentina. De igual manera, Sol (2015) en una muestra de niños argentinos concluyó que las fortalezas de la virtud del coraje están correlacionadas con los cinco elementos del bienestar propuestos por Seligman (2011). 
Asimismo, Unda-Villafuerte \& LupanoPerugini (2019), también hallaron correlaciones significativas entre la virtud del coraje y el bienestar psicológico en adolescentes ecuatorianos matriculados en bachillerato.

Con base a los objetivos de investigación planteados se pueden enunciar las siguientes conclusiones: a) niveles medios de bienestar y virtud del coraje en la muestra de adolescentes tardíos; b) no existen diferencias estadísticamente significativas en bienestar psicológico y la virtud del coraje de acuerdo al sexo; c) existen correlaciones positivas, moderadas y significativas entre la virtud del coraje y el bienestar - cada una de sus dimensiones. Sin embargo, estas conclusiones deben ser tomadas con cautela debido a las limitaciones naturales de un estudio descriptivo con una muestra por conveniencia, afectando una posible generalización de los resultados y las inferencias generadas de los datos.

Con respecto a líneas futuras de trabajo se recomienda emplear procedimientos estadísticos que permitan conocer las relaciones causales, por ejemplo: ¿Cuánto poder predictivo tendría la virtud del coraje en algunas de las dimensiones del bienestar psicológico? También otra línea interesante de trabajo futuro sería medir la totalidad de las virtudes y fortalezas del carácter y determinar su posible poder predictivo en una muestra aleatorizada de adolescentes montevideanos. Asimismo, incluir en el diseño de investigación algunas variables consideradas psicopatológicas como depresión, ansiedad y comportamiento violento para conocer sus relaciones a nivel de asociaciones o causales con las virtudes y fortalezas del carácter en estudios locales montevideanos.

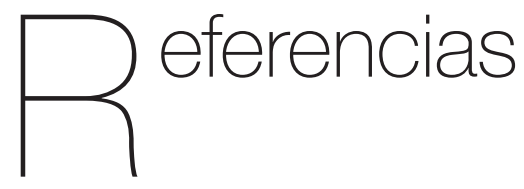

Abdel-Khalek, A. (2014). Happiness, health, and religiosity: significant associations among Lebanese adolescents. Mental Health, Religion \& Culture, 17(1), 30-38.

American Psychological Association. (2010). Manual de Publicaciones de la APA. Mexico: Editorial El Manual Moderno.

Amorín, D. (2008). Apuntes para una posible Psicología Evolutiva. Serie Cuadernos de Psicología Evolutiva, 1. Montevideo. Waskala Libros.

Brdar, I., \& Kashdan, T. (2010). Character strengths and well-being in Croatia: An empirical investigation of structure and correlates. Journal of Research in Personality, 44(1), 151-154.

Castellá, J., Saforcada, E., Tonon, G., Rodríguez, L., Mozobancyk, S., y Bedin, L. (2012). Bienestar subjetivo de los adolescentes: un estudio comparativo entre Argentina y Brasil. Psychosocial Intervention, 21, 273-280.

Castro-Solano, A. (2011). Las rutas de acceso al bienestar. Relaciones entre bienestar hedónico y eudaemónico. Un estudio en población argentina. Revista Iberoamericana de Diagnóstico y Evaluación Psicológica, 31(1), 37-57. 
Casullo, M. (2005). El capital psíquico. Aportes de la Psicología Positiva. Psicodebate, 6, 59-71.

Craig, G. J., \& Baucum, D. (2001). Desarrollo psicológico. México: Pearson Educación

Csikszentmihalyi, M. (1990). Fluir, una psicología de la felicidad. Barcelona: Kairos

Daset, L. R., Fernández-Pintos, M. E., Costa-Ball, D., López-Soler, C., \& Vanderplasschen, W. (2015). Desarrollo y validación del autoinforme de adolescentes: ADA. Ciencias Psicológicas, 9(1), 85-104

Duckworth, A. L., Peterson, C., Matthews, M. D., \& Kelly, D. R. (2007). Grit: Perseverance and passion for long-term goals. Journal of Personality and Social Psychology, 92, 1087-1101.

Duckworth, A., \& Gross, J. (2014). Selfcontrol and grit: Related but separable determinants of success. Current Directions in Psychological Science, 23(5), 319-325.

Duckworth, A., \& Seligman, M. (2006). Selfdiscipline gives girls the edge: Gender differences in self-discipline, grades, and achievement test scores. Journal of Educational Psychology, 98(1), 198-208.

Ejecutivo, U. P. (2008). Decreto 379/008. Investigación en Seres Humanos. Recuperado de http://www. elderechodigital. com. uy/smu/legisla D, 800379

Ferragut, M., Blanca, M.J. \& Ortiz-Tallo, M. (2014a). Analysis of adolescent profiles by gender: strengths, attitudes toward violence and sexism. Spanish Journal of Psychology, 17, 1-10.
Ferragut, M., Blanca, M.J., \& Ortiz-Tallo, M. (2014b). Psychological virtues during adolescence: A longitudinal study of gender differences. European Journal of Developmental Psychology, 11(5), 521-531

Fitermann, P., \&Zerpa, M. (2015). Aproximación al bienestar psicológico y la salud mental de los niños y adolescentes de Montevideo. En Cabella, W., Nathan, M., Fitermann, P., Vigorito, A., Zerpa, M., \& Bucheli, M. (eds.). Cambio familiar y bienestar de las mujeres y los niños en Montevideo y el área metropolitana: una perspectiva longitudinal (pp. 113-145). Montevideo: Universidad de la República y UNICEF.

Fredrickson, B. (2000). Handbook of Positive Psychology. New York: Oxford.

Garaigordobil, M., Aliri, J., \& Fontaneda, I. (2009). Bienestar psicológico subjetivo: diferencias de sexo, relaciones con dimensiones de personalidad y variables predictoras. Psicología Conductual, 17(3), 543-559.

García-Álvarez, D., Soler, M. J., \& Rendón, R. C. (2019). Bienestar psicológico en adolescentes: relaciones con autoestima, autoeficacia, malestar psicológico y síntomas depresivos. Revista de Orientación Educacional, 33(63), 23-43.

García-Andalete, J. (2013). Bienestar psicológico, edad y género en universitarios españoles. Salud \& Sociedad, 4(1), 48-58.

Giménez, M. (2010). La medida de las fortalezas psicológicas en adolescentes (VIAY): relación con clima familiar, psicopatología y bienestar psicológico (Tesis Doctoral de la Universidad Complutense de Madrid). Recuperado de https://www.tdx.cat/ handle/10803/516293 
Giménez, M., Vázquez, C., \& Hervás, G. (2010). El análisis de las fortalezas psicológicas en la adolescencia: Más allá de los modelos de vulnerabilidad. Psicología, Sociedad y Educación, 2, 83-100.

Gomez-Azcarate, E., Vera, A., Avila, M., Musitu, G., Vega, E. \& Dorantes, G. (2014). Resiliencia y felicidad de adolescentes frente a la marginación urbana en México. Psicodebate, 14(1), 45-68.

Góngora, V. \& Castro-Solano, A. (2014). Wellbeing and life satisfaction in Argentinean adolescents. Journal of Youth Studies, 17(9), 1277-1291.

Góngora, V., \& Castro-Solano, A. (2015). La validación de un índice de bienestar para población adolescente y adulta de la ciudad de Buenos Aires. PSIENCIA. Revista Latinoamericana de Ciencia Psicológica, 7, 329-338

González, R., Montoya, I., Casullo, M. \& Bernabéu, J. (2002). Relación entre estilos y estrategias de afrontamiento y bienestar psicológico en adolescentes. Psicothema, 14(2) 363-368.

Güemes-Hidalgo, M., González-Fierro, M. C., \& Vicario, M. H. (2017). Pubertad y adolescencia. Adolescere, 5(1), 7-22.

Hernández, R., Fernández, C. \& Baptista, P. (2008). Metodología de la investigación científica. México: Mc Graw Hill.

Huta, V., \& Hawley, L. (2010). Psychological strengths and vulnerabilities: Are they two ends of the same continuum or do they have independent relationships with wellbeing and ill-being? Journal of Happiness Studies, 11, 71-93.
Lyubomirsky, S. (2008). The how of happiness: A scientific approach to getting the life you want. New York: Penguin Press.

Miljković, D. \& Rijavec, M. (2008). What makes us happy: Strengths of mind, strengths of Herat or self-control? In M. Cindrić, V. Domović y M. Matijević (eds.), Pedagogy and the Knowledge Society (pp. 241-250). Zagreb: Ućiteljski fakultet

Organización Mundial de la Salud. (1995). The health of young people: a challenge and a promise. Ginebra: World Health Organization

Ovejero M., \& Cardenal, C. (2015). "Las fortalezas humanas desde la perspectiva de género: un estudio exploratorio en población española". Revista Mexicana de Investigación en Psicología, 7(2), 72-92.

Ovejero, M. (2010). Las fortalezas humanas desde la perspectiva de género. (Trabajo de fin de master). España: Universidad Complutense.

Papalia, D., Feldman, R. D. \& Martorell, G. (2012). Desarrollo humano. México: McGraw Hill.

Park, D., Tsukayama, E., Goodwin, G. P., Patrick, S., \& Duckworth, A. L. (2017). A tripartite taxonomy of character: Evidence for intrapersonal, interpersonal, and intellectual competencies in children. Contemporary Educational Psychology, 48, 16-27.

Park, N., Peterson, C., \& Seligman, M. (2004). Strengths of character and well-being. Journal of Social \& Clinical Psychology, 23, 603-619. 
Park, N., Peterson, C., \& Sun, J.K. (2013). La psicología positiva: Investigación y aplicaciones. Terapia Psicológica, 31, 11-19.

Peterson, C., \& Seligman, M. E. (2004). Character strengths and virtues: $A$ handbook and classification. New York, Oxford University Press.

Prieto, G., \& Muñiz, J. (2000). Un modelo para evaluar la calidad de los tests utilizados en España. Papeles del psicólogo, (77), 65-72

Quinlan, D., Vella-Brodrick, D. A., Gray, A., \& Swain, N. (2018). Teachers matter: Student outcomes following a strengths intervention are mediated by teacher strengths spotting. Journal of Happiness Studies, 1-17.

Rice, F. (1997). Desarrollo Humano. México: Prentice Hall Hispanoamericana

Sánchez-Hernández, Ó. (2015). Sobre las fortalezas humanas y las diferencias de género. Revista Mexicana de Investigación en Psicología, 7(2), 107-116.

Seligman, M. (2003). La auténtica felicidad. Barcelona: Vergara

Seligman, M. (2011). Flourish: A Visionary New Understanding of Happiness and Well-being. New York: Free Press.

Seligman, M., Steen, T., Park, N., \& Peterson, C. (2005). Positive psychology progress: Empirical validation of interventions. American Psychologist, 60, 410-421.

Shankland, R., \& Rosset, E. (2017). Review of brief school-based positive psychological interventions: A taster for teachers and educators. Educational Psychology Review, 29(2), 363-392.

Shimai, S., Otake, K., Park, N., Peterson, C. \& Seligman, M. E. P. (2006). Convergence of character strengths in American and Japanese young adults. Journal of Happiness Studies, 7, 311-322.

Sol, A. (2012). El análisis de las virtudes y fortalezas en niños: una breve revisión teórica. Psicodebate. Psicología, Cultura y Sociedad, 12, 39-61.

Sol, A. (2015). El estudio de las fortalezas del carácter en niños: Relaciones con el bienestar psicológico, la deseabilidad social y la personalidad. Psicodebate. Psicología, Cultura y Sociedad, 15(1), 43-68.

Soler, M. (2016). Virtud del coraje en relación al bienestar psicológico en adolescentes (Tesis Doctoral inédita de la Universidad de Flores). Buenos Aires, Argentina.

Unda-Villafuerte, F. \& Lupano-Perugini, M. (2019). Virtuousness profiles in school organizations in relations to psychological well-being levels in Ecuadorian adolescents. Revista Cátedra, 2(2), 76-93.

Vargas, J. (2009). Percepción de clima social familiar y actitudes ante situaciones de agravio en la adolescencia tardía Interdisciplinaria, 26(2), 289-316.

Wagner, L. (2019). Good character is what we look for in a friend: character strengths are positively related to peer acceptance and friendship quality in early adolescents. The Journal of Early Adolescence, 39(6), 864-903. 
Weber, M., Wagner, L., \& Ruch, W. (2016). Positive feelings at school: On the relationships between students' character strengths, school-related affect, and school functioning. Journal of Happiness Studies, 17, 341-355.
Zubieta, M., Muratori, M. \& Fernández, D. (2012). Bienestar subjetivo y psicosocial: explorando diferencias de género. Salud \& Sociedad, 3(1), 66-76. 